\title{
Infecção urinária relacionada a cateterismo vesical de demora: Pesquisa bibliográfica
}

\author{
Urinary infection related to delayed bladder probe: Bibliographic research \\ Infección urinaria relacionada con sonda de vejiga: Investigación bibliográfica
}

Recebido: 16/06/2021 | Revisado: 22/06/2021 | Aceito: 24/06/2021 | Publicado: 10/07/2021

Carolina Martins dos Santos

ORCID: https://orcid.org/0000-0003-3316-0409

Centro Universitário de Volta Redonda, Brasil

E-mail: carolina.rinaldi@ hotmail.com

Laís da Costa Campos

ORCID: https://orcid.org/0000-0002-1203-6364

Centro Universitário de Volta Redonda, Brasil E-mail: laiscampos.173@gmail.com

Thalita Cristina de Carvalho Raimundo

ORCID: https://orcid.org/0000-0003-0704-0518

Centro Universitário de Volta Redonda, Brasil

E-mail: thalitacarvalho-vr1@ hotmail.com

Clarissa Ferreira Pontual de Oliveira

ORCID: https://orcid.org/0000-0002-2915-9205

Centro Universitário de Volta, Brasil

E-mail: pontualclarissa@gmail.com

Renata Martins da Silva Pereira

ORCID: https://orcid.org/0000-0001-7642-6030

Centro Universitário de Volta Redonda, Brasil

Universidade do Estado do Rio de Janeiro, Brasil

E-mail: renataenfprofessora@gmail.com

\begin{abstract}
Resumo
Este estudo teve como objetivo principal: apontar os fatores que influenciam no surgimento de infecções urinárias após a instalação de cateterismo vesical de demora. Pesquisa bibliográfica, exploratória, descritiva, com uma abordagem qualitativa. O levantamento dos artigos foi realizado na Biblioteca Virtual em Saúde (BVS) e Google Acadêmico que integra as seguintes bases de dados: Lilacs, BDENF e Scielo. Foram utilizados como descritores: "Infecção Urinária", "Cateterismo Vesical de Demora" e "Enfermagem". O período de publicação foi delimitado entre os anos de 2007 a 2018. A coleta de dados deu-se em abril 2020. A análise dos artigos permitiu formular 4 categorias temáticas: tempo de permanência do cateter, altos índices de infecção urinária em UTI, condições assépticas para a realização do procedimento e fatores de risco associados a instalação de infecção urinária pós cateterismo vesical de demora. Os resultados nos permitiram observar que, o tempo de permanência do cateter no sistema urinário contribui para o surgimento de infecção urinária após o cateterismo vesical de demora. Ainda foi possível perceber que, são altos os índices de infecção urinária por este procedimento em Unidades de Terapia Intensiva e que os enfermeiros necessitam em todos os cenários hospitalares estarem preparados técnicocientificamente para a realização desse cuidado junto ao paciente de forma asséptica e estéril. Concluiu-se que a infecção do trato urinário consiste em um importante problema de saúde pública na atualidade e que pode surgir após o procedimento cateterismo vesical de demora, sendo mister o emprego de técnica correta e asséptica para sua condução.
\end{abstract}

Palavras-chave: Infecção urinária; Cateterismo vesical de demora; Enfermagem.

\begin{abstract}
This study had as main objective: to point out the factors that influence the emergence of urinary infections after the installation of indwelling vesical catheterization. Bibliographic research, exploratory, descriptive, with a qualitative approach. The survey of articles was carried out in the Virtual Health Library (BVS) and Academic Google, which integrates the following databases: Lilacs, BDENF and Scielo. The following descriptors were used: "Urinary Infection", "Delayed Vesical Catheterization" and "Nursing". The period of publication was delimited between the years 2007 to 2018. Data collection took place in April 2020. The analysis of the articles allowed the formulation of 4 thematic categories: length of catheter permanence, high rates of urinary infection in the ICU, conditions aseptic for the procedure and risk factors associated with the installation of urinary infection after indwelling vesical catheterization. The results allowed us to observe that the permanence time of the catheter in the urinary system contributes to the emergence of urinary infection after indwelling urinary catheterization. It was also possible to
\end{abstract}


notice that the rates of urinary infection by this procedure in Intensive Care Units are high and that nurses need, in all hospital scenarios, to be technically-scientifically prepared to carry out this care with the patient in an aseptic and sterile manner. It was concluded that urinary tract infection is an important public health problem today and that it can arise after the indwelling vesical catheterization procedure, requiring the use of correct and aseptic technique for its management.

Keywords: Urinary infection; Delayed vesical catheterization; Nursing.

\section{Resumen}

Este estudio tuvo como principal objetivo: señalar los factores que influyen en la aparición de infecciones urinarias tras la instalación de cateterismo vesical permanente. Investigación bibliográfica, exploratoria, descriptiva, con enfoque cualitativo. La encuesta de artículos se realizó en la Biblioteca Virtual en Salud (BVS) y Google Académico, que integra las siguientes bases de datos: Lilacs, BDENF y Scielo. Se utilizaron los siguientes descriptores: "Infección urinaria", "Sondaje vesical tardío" y "Enfermería". El período de publicación se delimitó entre los años 2007 a 2018. La recolección de datos se realizó en abril de 2020. El análisis de los artículos permitió la formulación de 4 categorías temáticas: tiempo de permanencia del catéter, altas tasas de infección urinaria en la UCI, condiciones asépticas para el procedimiento y los factores de riesgo asociados con la instalación de una infección urinaria después de un cateterismo vesical permanente. Los resultados nos permitieron observar que el tiempo de permanencia del catéter en el sistema urinario contribuye a la aparición de infección urinaria tras el cateterismo urinario permanente. También se pudo notar que las tasas de infección urinaria por este procedimiento en las Unidades de Cuidados Intensivos son altas y que el enfermero necesita, en todos los escenarios hospitalarios, estar preparado técnico-científico para realizar este cuidado con el paciente en forma aséptica y estéril. manera. Se concluyó que la infección del tracto urinario es un importante problema de salud pública en la actualidad y que puede surgir después del procedimiento de cateterismo vesical permanente, requiriendo el uso de una técnica correcta y aséptica para su manejo.

Palabras clave: Infección urinaria; Cateterismo vesical retardado; Enfermería.

\section{Introdução}

Este estudo trata-se de uma pesquisa bibliográfica acerca do surgimento de sinais e sintomas da infecção urinária em pacientes submetidos ao cateterismo vesical de demora e o papel da enfermagem.

O interesse pelo estudo surgiu após experiências profissionais de uma das autoras como técnica em enfermagem, onde foi possível perceber o alto índice de infecções urinárias em pacientes que foram submetidos ao cateterismo vesical de demora. Segundo Ferreira, et al. (2017), 80\% dos casos de infecção do trato urinário desenvolvido no período de internação estão relacionados a presença do cateter vesical de demora. Desta forma, surgiu a necessidade de conhecer o papel da equipe de enfermagem na detecção e prevenção da infecção urinária nesse contexto.

O cateterismo vesical de demora (CVD) é compreendido como a introdução de uma sonda, que irá alcançar a bexiga, após uma introdução uretral ou pela via suprarrenal tendo duração de dias, ou meses. (Ercole et al., 2013).

Este procedimento é utilizado em situações específicas, e sua prescrição tem o objetivo de esvaziar a bexiga para finalidades cirúrgicas e/ou diagnósticas, e em casos de patologias diversas. Pode ser realizada também junto a esse procedimento a administração de medicamentos, além de permitir uma avaliação exata do débito urinário do paciente. Seus principais riscos estão relacionados com as infecções do trato urinário e traumatismos na uretra, ocasionados por forçar o cateter na uretra no momento da introdução do mesmo. (Godoy, 2015).

Os principais agente etiológicos causadores de infecção urinária são as bactérias; as infecções fúngicas e virais são incomuns. Sendo que em $80 \%$ dos casos a ITU é provocada pela bactéria Escherichia coli. Outros microrganismos prevalentes são Klebsiella, Proteus, Enterobacter, Citobacter e Pseudomonas. É mais prevalente no sexo feminino. São muito comuns e responsáveis pela maioria dos processos infecciosos hospitalares. A ITU é caracterizada pela presença de micro-organismos nas vias urinárias, sendo no trato urinário, na bexiga, próstata, sistema coletor ou rins. Seu surgimento resulta em grande repercussão econômica, devido ao tratamento que será ofertado ao paciente, gerando custos extras de internação, além de grandes potenciais de complicações que podem ocorrer ocasionando danos e/ou sequelas ao paciente (Marks, et al. 2020).

Os principais fatores de risco são: sexo feminino, idade avançada, manipulação do trato urinário, disfunções anatômicas e fisiológicas do sistema, patologias associadas e tempo de duração do cateterismo (Oliveira et al., 2008). 
De acordo com os conhecimentos da microbiologia a Escherichia coli é a mais recorrente, no caso de infecção que são contraídas em comunidade a sua prevalência é de $80 \%$ e caso o paciente esteja internando sua contração é de 50 a $60 \%$, ainda falando das infecções adquiridas em comunidade o Staphylococcus saprophyticus são representatividade e em sua grande maioria é em mulheres jovens e sexualmente ativas e sua prevalência de contração no hospital é de 3,9\%, Proteus spp e Kleibsiella spp são de baixa prevalência ocorrendo entre 10 à 15\%, nas classes de bactérias de infecção no trato urinário em meio hospitalar são a Escherichia coli, Pseudomonas aeruginosa, Enterococcus faecalis (Rodrigues \& Barroso, 2011).

Os sinais e sintomas das infecções urinárias devido ao cateterismo vesical de demora não fogem muito da realidade de uma ITU que encontramos no dia a dia, sendo elas: hematúria, urina turva, urgência urinária, polaciúria, disúria, oligúria, dor no abdome inferior, nictúria, urina com odor fétido ou desagradável, além da possibilidade de febre e desconforto que também podem ocorrer. (Griffin \& Potter, 2015)

Neste contexto, a enfermagem deverá utilizar de seus conhecimentos técnico-científicos para realização de cuidados visando diminuir os riscos de ITU, dentre eles: lavagem das mãos de forma adequada, higienização corporal diária do paciente, monitorização das técnicas que estão sendo realizadas. Além disso, torna-se necessário conscientizar os membros da equipe de enfermagem quanto a importância dos treinamentos em serviço para aprimorar cada vez mais a realização do procedimento e a implantação de protocolos de intervenções para serem seguidos de forma unificada.

Um estudo feito em análises de prontuários com pacientes admitidos em uma Unidade de Terapia Intensiva mostrou que o cateterismo vesical de demora usado em grande escala sem indicação e por um longo tempo, ofereceu riscos iminentes para infecção urinária no paciente. (Mota \& Oliveira, 2019).

Ao descrever os cuidados de enfermagem associados à prevenção de infecção devido ao cateterismo vesical de demora na Unidade de Terapia Intensiva (UTI), percebe-se que as intervenções necessitam ser realizadas de maneira correta para minimizar o índice de infecções, tendo como sugestão a utilização de protocolos baseados em pesquisas cientificas. (Cardoso \& Maia, 2014)

Algumas medidas, como: visitas diárias da equipe multidisciplinar revisando a necessidade da manutenção do cateter, desenvolvimento de programa o qual possa se identificar e remover cateteres desnecessários, monitoramento dos eventos adversos, como obstrução do cateter, remoção acidental, dentre outras, são ações que podem contribuir para redução de infecções associadas ao cateterismo vesical de demora (Silva; Souza et al., 2016).

Estabeleceu-se como questão a investigar do estudo:

- O que a produção científica revela acerca do surgimento da infecção urinária após o procedimento cateterismo vesical de demora e os cuidados de enfermagem?

Para responder essa questão, traçou-se como objetivo principal da pesquisa:

- Apontar os fatores que influenciam significativamente no surgimento de infecções urinárias após a instalação de cateterismo vesical de demora, descritos nas produções bibliográficas.

\section{Metodologia}

O estudo realizado consiste em uma pesquisa bibliográfica, exploratória, descritiva, com uma abordagem qualitativa. Michel (2015) define a pesquisa qualitativa, como aquela que se propõe a colher e analisar dados descritivos, obtidos diretamente da situação estudada; enfatiza o processo mais que o resultado, para o que precisa e retrata a perspectiva dos participantes. Na pesquisa qualitativa, verifica-se a realidade em um contexto natural, tal como ocorre na vida real, procurando dar sentido aos fenômenos ou interpretá-los, de acordo com os significados que possuem para as pessoas implicadas nesse contexto (Michel, 2015). 
O levantamento dos artigos foi realizado na Biblioteca Virtual em Saúde (BVS) e Google Acadêmico que integra as seguintes bases de dados: Literatura Latino-Americana e do Caribe em Ciências da Saúde (Lilacs), Base de Dados de Enfermagem (BDENF) e Scientific Electronic Library Online (Scielo).

A estrutura da pesquisa em cada base de dados foi conduzida por meio dos descritores controlados pelo DeCS (Descritores em Saúde): "Infecção Urinária", “Cateterismo Vesical de Demora" e "Enfermagem”, cruzado entre si e em todas as possibilidades. Foram encontrados 11 artigos e utilizamos os seguintes filtros: texto completo e disponível, bases de dados supracitadas e idioma português. Desses, 01 apareceu em duplicidade. Portanto, foram analisados 10 artigos publicados entre os anos de 2007 a 2018.

A etapa de seleção dos estudos envolveu a leitura crítica e atenta dos artigos na íntegra, aplicando os seguintes critérios: 1) Inclusão - estudos originais, publicados no idioma português, que abordassem o surgimento da infecção urinária em pacientes submetidos ao cateterismo vesical de demora. 2) Exclusão - não atendesse aos critérios de inclusão e estarem duplamente indexados nas bases. A coleta de dados deu-se no período do mês de abril de 2020.

Inicialmente, fez-se uma leitura flutuante dos artigos selecionados, e logo em seguida, realizou-se uma leitura analítica dos artigos, sucedendo na interpretação dos dados. Após a interpretação dos dados, fez-se possível construir categorias temáticas.

A análise dos artigos selecionados permitiu identificar a visão de diferentes pesquisadores, e posteriormente, agrupálas de acordo com as semelhanças. Formulamos, assim, 4 categorias para discutir as diferentes abordagens dos trabalhos: tempo de permanência do cateter, altos índices de infecção urinária em UTI, condições assépticas para a realização do procedimento e fatores de risco associados a instalação de infecção urinária pós cateterismo vesical de demora.

\section{Resultados e Discussão}

Como resultado obteve-se: 11 artigos das bases de dados Scielo, Lilacs e BDENF. Selecionamos apenas os artigos com a possibilidade de acessar o texto completo on line, em português. Destes, 01 apareceu em duplicidade.

Quadro 1 - Distribuição dos estudos sobre o surgimento da infecção urinária em pacientes submetidos ao cateterismo vesical e a enfermagem, segundo título, revista, ano e objetivos. Volta Redonda/ RJ, 2020.

\begin{tabular}{|c|c|c|c|}
\hline Título & Revista & Ano & Objetivos \\
\hline $\begin{array}{l}\text { Pós - Operatório de } \\
\text { Vulvectomia e Cateterismo } \\
\text { Vesical de Demora: Revisão } \\
\text { Integrativa. }\end{array}$ & $\begin{array}{l}\text { Revista de } \\
\text { Enfermagem - UFPE } \\
\text { On line }\end{array}$ & 2017 & $\begin{array}{l}\text { Identificar os benefícios e riscos relacionados ao uso do } \\
\text { Cateterismo Vesical de Demora no pós-operatório de } \\
\text { vulvectomia em oncologia. }\end{array}$ \\
\hline $\begin{array}{l}\text { Prevenção de Infecção } \\
\text { Urinária: Indicadores de } \\
\text { qualidade da Assistência de } \\
\text { Enfermagem em Idosos. }\end{array}$ & $\begin{array}{l}\text { Revista de } \\
\text { Enfermagem - UFPE } \\
\text { On line }\end{array}$ & 2017 & $\begin{array}{l}\text { Analisar a assistência de Enfermagem, a partir de } \\
\text { indicadores, com foco na prevenção da infecção urinária. }\end{array}$ \\
\hline $\begin{array}{l}\text { Incidência de infecção urinária } \\
\text { em receptores de transplante } \\
\text { renal. }\end{array}$ & $\begin{array}{l}\text { Acta Scientiarum. } \\
\text { Health Sciences }\end{array}$ & 2017 & $\begin{array}{l}\text { Investigar o número de infecções urinárias em pacientes } \\
\text { transplantados renais, visando à melhoria dos cuidados de } \\
\text { enfermagem a fim de amenizar a incidência de infecção } \\
\text { urinária. }\end{array}$ \\
\hline
\end{tabular}




\begin{tabular}{|c|c|c|c|}
\hline $\begin{array}{l}\text { Controle de Infecção em } \\
\text { cateterismo Vesical de Demora } \\
\text { em Unidade de Terapia } \\
\text { Intensiva }\end{array}$ & $\begin{array}{lr}\text { Revista } & \text { de } \\
\text { Enfermagem } & \text { do } \\
\text { Centro } & \text { Oeste } \\
\text { Mineiro } & \end{array}$ & 2015 & $\begin{array}{l}\text { Avaliar as práticas de controle e prevenção de infecções do } \\
\text { trato urinário, no uso de cateter vesical numa unidade de } \\
\text { Terapia Intensiva geral de um hospital da grande } \\
\text { Florianópolis/Santa Catarina. }\end{array}$ \\
\hline $\begin{array}{l}\text { Cateterismo urinário: } \\
\text { conhecimento e adesão ao } \\
\text { controle de infecção pelos } \\
\text { profissionais de enfermagem; }\end{array}$ & $\begin{array}{l}\text { Revista Eletrônica de } \\
\text { Enfermagem }\end{array}$ & 2007 & $\begin{array}{l}\text { Identificar o conhecimento e a adoção das medidas } \\
\text { recomendadas para prevenção e controle de infecção no } \\
\text { manuseio e instrumentação do trato urinário associado a } \\
\text { cateter vesical pelos profissionais de enfermagem e } \\
\text { verificar a existência de rotina escrita e capacitação em } \\
\text { serviço. }\end{array}$ \\
\hline $\begin{array}{l}\text { Infecção do trato urinário } \\
\text { associada a cateter vesical: por } \\
\text { que não controlamos esse } \\
\text { evento adverso }\end{array}$ & $\begin{array}{l}\text { Revista da escola de } \\
\text { Enfermagem da USP. }\end{array}$ & 2018 & $\begin{array}{l}\text { Identificar fatores relacionados à ocorrência de infecção do } \\
\text { trato urinário associado ao uso de cateter urinário. }\end{array}$ \\
\hline $\begin{array}{l}\text { Ações de enfermagem para } \\
\text { prevenção de infecção do trato } \\
\text { urinário relacionada ao cateter } \\
\text { vertical de demora }\end{array}$ & Revista Einsten & 2009 & $\begin{array}{l}\text { Estudar as ações de enfermagem que previnem a infecção do } \\
\text { trato urinário relacionado ao cateter vertical de demora e } \\
\text { descrever a importância das ações intervencionistas de } \\
\text { enfermagem na redução da incidência de infecção do trato } \\
\text { urinário relacionada ao cateter vesical de demora, em } \\
\text { pacientes clinicamente enfermos internados em UTI. }\end{array}$ \\
\hline $\begin{array}{l}\text { Infecção do trato urinário } \\
\text { relacionado com uso do cateter: } \\
\text { revisão integrativa }\end{array}$ & $\begin{array}{l}\text { Revista } \\
\text { enfermagem } \\
\text { Referência }\end{array}$ & 2013 & $\begin{array}{l}\text { Realizar a revisão integrativa da literatura para identificar } \\
\text { evidências científicas que relacionam o cateter urinário de } \\
\text { alívio, intermitente e de demora com a infecção do trato } \\
\text { urinário }\end{array}$ \\
\hline $\begin{array}{l}\text { Incidência de infecção do trato } \\
\text { urinário relacionada ao } \\
\text { cateterismo vertical de demora: } \\
\text { um estudo de coorte. }\end{array}$ & $\begin{array}{l}\text { Revista Mineira de } \\
\text { enfermagem }\end{array}$ & 2016 & $\begin{array}{l}\text { Analisar os aspectos epidemiológicos das ITU em pacientes } \\
\text { submetidos ao CVD internados em Centros de Terapia } \\
\text { Intensiva (CTI) de dois hospitais de Belo Horizonte }\end{array}$ \\
\hline $\begin{array}{l}\text { Cuidados de enfermagem para } \\
\text { prevenção de infecção do trato } \\
\text { urinário em pacientes com } \\
\text { cateterismo vesical de } \\
\text { demora(CVD) no ambiente } \\
\text { hospitalar. }\end{array}$ & $\begin{array}{l}\text { Arquivos médicos dos } \\
\text { hospitais e da } \\
\text { faculdade de ciências } \\
\text { médicas da Santa casa } \\
\text { de São Paulo }\end{array}$ & 2018 & $\begin{array}{l}\text { Identificar na literatura os cuidados de enfermagem para } \\
\text { prevenção de infecção do trato urinário em pacientes com } \\
\text { cateterismo vertical, no ambiente hospitalar. }\end{array}$ \\
\hline
\end{tabular}

Fonte: Autores (2021).

\section{Tempo de Permanência do Cateter}

Após a análise dos artigos científicos, tornou-se viável a percepção do tempo de permanência do cateter no sistema urinário, pois é um fator importante e que contribui, consideravelmente, para o surgimento de infecção urinária após o procedimento cateterismo vesical de demora. 
Além disso, na teoria de enfermagem de Vírginia Henderson (1969), a prática da enfermagem profissional significa a atuação para compensação de qualquer ato, na observação, no cuidado e no aconselhamento dos doentes, feridos ou inválidos; na manutenção da saúde ou prevenção da doença de outros; exigindo julgamento especializado substancial e habilidade baseados no conhecimento e na aplicação dos princípios das ciências biológicas, físicas e sociais. (American Nurses Association, 2009).

O enfermeiro é um profissional vital nesse processo, pois junto com a equipe do Controle de Infecção Hospitalar $(\mathrm{CCIH})$ estabelecerá protocolos a serem seguidos, incluindo o tempo em que o cateter deve permanecer no organismo do paciente. Em conjunto com a equipe interdisciplinar de saúde, será avaliado se o paciente necessita continuar com o cateterismo, culminando, assim, possibilidade de realizar um novo procedimento, ou não.

Os trechos abaixo revelam uma relação diretamente proporcional entre tempo de permanência do cateterismo vesical de demora e o diagnóstico de infecção do trato urinário:

Estudos demostram um risco de 2,5\% para um dia de cateterismo, 10\% para dois a três dias, 12,2\% para quatro a cinco dias, chegando a 26,9\% com duração igual ou maior do que seis dias. Artigo 8

Aproximadamente $80 \%$ das ITU's relacionadas à assistência à saúde estão associadas ao uso do cateter vesical $(\mathrm{CV})$. O risco de desenvolver ITU's associada a CV aumenta com duração do cateterismo, podendo chegar a 5\% a cada dia de uso. Assim, estima-se que, após 28 dias de cateterização, esse risco eleve-se para 100\%, culminando em aproximadamente $4 \%$ dos pacientes com evolução para sepse secundária à infecção e uma taxa de mortalidade estimada em até $30 \%$. Artigo 7

A duração do cateterismo é, provavelmente, o mais importante fator de risco na instalação da bacteriúria associada ao cateter de demora. Estudos epidemiológicos têm demonstrado claramente que o risco de infecção urinária, associada ao cateter de demora, está relacionado com a duração do cateterismo. Tem sido encontrada uma progressiva bacteriúria em pacientes com cateter de demora. Assim, foi verificado que, quando a contagem microbiana era inicialmente mais baixa detectável em 90\%dos casos ocorreu um aumento para 105 colônias/ ml de urina, dentro de três dias. Artigo 4

Desta forma, o tempo como alto risco deve ser limitado ao mínimo possível com a sonda para que o índice de ITU não ocorra com tanta frequência e, consequentemente, o profissional enfermeiro necessita estar com respaldos científicos e/ou protocolos para saber a importância de se atenuar ao tempo de permanência do cateter e para que ele possa dar uma assistência de qualidade para seus pacientes e, assim, administrar o seu setor com excelência. (Pereira, et al. 2014). Corroborando com essas ideias, surge ainda o trecho abaixo:

O índice de $100 \%$ de não conformidade de indicação de CV é demonstrado pela ausência de registros no prontuário quanto à indicação, cuidados prestados e tempo de permanência. Estabelecendo critérios (protocolo) para o CVD e tempo de permanência, realizando a retirada em tempo adequado do $\mathrm{CV}$, diminuem as intervenções e probabilidade de infecções. Artigo 4

Caetano e Maia (2014), destacam a importância de os membros da equipe de enfermagem estarem se capacitando continuamente para compreender essa relação entre tempo de permanência do cateter e o surgimento dos sinais e sintomas de 
infecção do trato urinário.

\section{Altos Índices de infecção urinária em UTI}

A análise dos artigos pesquisados possibilitou-nos, ainda, identificar os casos de ITU nas Unidades de Terapia Intensiva que são significativamente maiores do que em outros setores hospitalares, como é possível observar nos trechos abaixo:

As infecções são manifestações frequentes na Unidade de Terapia Intensiva (UTI) devido à gravidade do paciente, maior diversidade microbiana e maior exposição a procedimentos invasivos como cateterismo vesical, indicado na maioria das vezes para avaliação do débito urinário, e em pacientes comatosos e sedados. Artigo 8

A UTI recebe pacientes graves, em situação de risco que buscam nesse local de internação condições de sobrevida, entretanto a UTI é o local de maior probabilidade de um paciente adquirir infecção. Artigo 4

A UTI é uma área no hospital onde o risco de aquisição, de infecção hospitalar é particularmente elevado, pelas seguintes razões:

- os pacientes de UTI estão gravemente doentes e geralmente possuem mais de uma doença em curso comparado com os outros pacientes.

- procedimentos invasivos Como cateterismo vesical são mais realizados na UTI.

- o uso excessivo de antibióticos de amplo espectro provocam formação de micro-organismos resistentes que, por fim, favorecem o surgimento de infecção.

- o ritmo das atividades na UTI pode, em geral, tornar a equipe de enfermagem e demais profissionais de saúde menos diligentes com a técnica asséptica. Artigo 8

As Unidades de Terapia Intensiva se caracterizam pela internação de pacientes criticamente enfermos que necessitam de acompanhamento específico 24 horas por dia por parte de uma equipe interdisciplinar de saúde tecnicamente preparada para oferecer uma assistência de qualidade, livre de riscos e danos à saúde do paciente.

Em estudo realizado em Belém (PA) destacou que os dispositivos invasivos mais utilizados na UTI foram as sondas (sonda vesical de demora/ sonda vesical de alívio), seguindo do uso de cateter venoso central e do ventilador mecânico. Diante deste resultado, foi possível observar uma elevação na ocorrência de infecções relacionadas à assistência a saúde (IRAS) em pacientes que foram submetidos a realização de um grande número de procedimentos invasivos destacados como fatores de risco para mortalidade, porém não foram encontrados dados suficientes para a elaboração de uma correlação significante entre a utilização de qualquer dispositivo mencionado ao aumento da aquisição de IRAS (Trindade, et al., 2020).

Cerca de 20 a 30\% dos casos de IRAS no Brasil, são acometidas nas Unidades de Terapia Intensiva (UTI) e, dentre os números, as infecções do trato urinário (ITU’s), associadas ao cateterismo vesical, chegam a atingir 10 a $30 \%$ dos pacientes internados nestas condições, sendo apontadas como uma importante causa das taxas de morbimortalidade deste setor hospitalar. (Farias, Nascimento \& Souza, 2019).

As infecções do trato urinário (ITU’s) necessitam de maior atenção por parte da equipe de saúde que atua na UTI e nos outros setores hospitalares, pois se trata da infecção hospitalar mais comum e com alta incidência. (Menegueti; Gonçalves et. al., 2012). O artigo abaixo destaca ainda essa questão: 
É um destaque dentre as infecções, a incidência daquelas relacionados ao trato urinário, correspondendo entre 35\% a $40 \%$ de todas as infecções hospitalares. Sendo $70 \%$ a $88 \%$ diretamente relacionadas ao cateterismo vesical e 5\% a $10 \%$ após cistoscopias ou procedimentos cirúrgicos com manuseio do trato urinário, sobretudo em ambientes como UTI's e Semi- UTI's. Artigo 2

Portanto, a equipe de enfermagem necessita estar preparada técnico-cientificamente para a assistência de pacientes em qualquer cenário do cuidado, incluindo as Unidades de Terapia Intensiva. Esse setor, todavia, apresenta peculiaridades significativas para o cuidado em saúde, uma vez que o paciente que se encontra internado possui maiores necessidades em relação ao tratamento oferecido, perecendo de vital importância, estratégias para a redução dos riscos de contrair uma infecção devido ao procedimento cateterismo vesical de demora.

\section{Condições Assépticas para Realização do Procedimento}

Emergiram ainda na presente pesquisa, trechos de artigos científicos que destacaram as condições assépticas para realização do cateterismo vesical de demora, como visto nos parágrafos abaixo:

Foram identificadas evidências, nos estudos, associados aos cuidados de Enfermagem na prevenção da ITU, sendo fundamental que estes ultrapassem a técnica. Os riscos devem ser constantemente avaliados; a prática de higienização das mãos deve ser realizada criteriosamente; as condições anatômicas de cada paciente devem sempre ser levadas em consideração na hora da fixação e manipulação, assim como a educação do paciente e de seus familiares. Artigo 3

$O$ treinamento da equipe de Enfermagem consiste em capacitar auxiliares e técnicos de Enfermagem e executarem a técnica de cateterismo vesical de forma asséptica, educando-os quanto à lavagem das mãos, visto que estes são os principais responsáveis pelas infecções cruzadas e pelos surtos de ITU nas UTI's, revelando a necessidade de uma boa higienização no atendimento a um paciente. Artigo 8

A implementação e avaliação de programas e protocolos de controle da infecção em UTI deve ser continuo, pois proporcionam prestação de cuidados ao paciente de forma adequada. A capacitação das equipes resulta na intensificação da vigilância para a diminuição de falhas relativas à higiene intima do paciente, desinfecção após desprezar a diurese. Na identificação dos dispositivos e anotações documentais dos procedimentos. Artigo 4

Dentre os profissionais que compõe a equipe de enfermagem, fica sob a responsabilidade do enfermeiro a realização de procedimentos invasivos, como CVD. Para isso, o profissional deve realizar esse procedimento de forma asséptica sem contaminar nenhuma etapa do cuidado oferecido. Destaca-se, portanto, que esse procedimento tem que ser realizado de forma estéril.

A introdução do cateter nesse procedimento precisa ser feita pelo enfermeiro. A retirada do cateter pode ser realizada por todos os membros da equipe de enfermagem. Para tanto, esses profissionais necessitam de treinamento em serviço para ser realizado sem contaminação, livre de riscos e danos à saúde dos pacientes.

O papel da equipe de enfermagem é essencial para a prevenção de infecções, principalmente relacionadas as ITU`s, as quais os profissionais devem buscar desenvolver práticas baseadas em evidências, resultando, dessa maneira, em menores índices, diminuindo as ocorrências, elevando a qualidade do serviço. (Ercole et al.,2013). Destaca-se o trecho abaixo: 
Destaca-se também que a inserção do CV, definida pelo parecer normativo do COFEN, "é atividade que necessita de profissionais treinados e habilitados, por se tratar de procedimento invasivo, que envolve riscos ao paciente". Ainda, "requer cuidado de maior complexidade técnica, conhecimentos de base cientifica e, por essas razões, no âmbito da equipe de enfermagem, a inserção de CV é privativa do enfermeiro”, o que reforça sua participação na avaliação, desde sua indicação até a manutenção diária, bem como nas práticas adotadas de sua manipulação. Artigo 7

O enfermeiro, portanto, fica responsável pela inserção do cateter do procedimento cateterismo vesical de demora, e deve realizar o cuidado de modo a prevenir complicações futuras. É de suma importância ressaltar que os demais cuidados relacionados à avaliação, manutenção e retirada do cateter cabe a todos os membros da equipe de enfermagem. A equipe necessita estar treinada para estabelecer estratégias de prevenção de infecção do trato urinário, identificação precoce dos sinais e sintomas.

\section{Fatores de Risco associados a instalação de infecção urinária pós cateterismo vesical de demora}

Os artigos científicos revelaram que alguns fatores de risco possibilitam o aparecimento de infecções no trato urinário após a cateterização vesical de demora, como observa-se abaixo:

A idade avançada, sexo feminino, disfunções anatômicas e doenças subjacentes severas estão entre os principais fatores de risco associados às ITU (Infecções do Trato Urinário). Mas, sem dúvida, a grande maioria destas infecções, cerca de 80\%, está associada ao cateterismo do trato urinário, sua duração, manipulação, posicionamento e garantia de fluxo. Artigo 3

Vários são os fatores de risco responsabilizados pela alta prevalência da bacteriúria associada ao cateter de demora. Entre eles, estão incluídos o sexo, a idade avançada e doença grave coexistente. A idade avançada e a coexistência de uma doença grave, em pacientes cateterizados e hospitalizados, constituem importantes fatores de risco que proporcionam, inclusive, um aumento da taxa de mortalidade. Artigo 3

Esse risco depende tanto de fatores predisponentes relacionados ao paciente como: sexo feminino, idade avançada, diabetes, imunocomprometimento, e fatores externos associados principalmente, a iatrogenia no manuseio, com quebra da assepsia e longo tempo de permanência do cateter. Artigo 6

Quanto aos fatores sociodemográficos e clínicos do paciente que podem aumentar o risco de ITU-CV, idade avançada e sexo feminino são considerados grupos com maiores predisposição. Porém, neste estudo, as taxas de ITU-CV estiveram associadas a fatores clínicos, como o tempo de permanência do CV e do paciente na UTI. Artigo 7

Os principais fatores de risco para o surgimento das infecções relacionadas a assistência à saúde estão relacionados à idade, presença de doenças crônicas degenerativas (diabetes e neoplasias); realização de procedimentos incorretos; erros durante antissepsia da pele e na esterilização de materiais; circulação de várias pessoas no ambiente restrito, uso incorreto de antimicrobianos; bem como a não higienização correta por parte dos profissionais (Trindade et al., 2020).

Em síntese, pacientes com o uso do cateter urinário têm o risco maior de adquirir uma ITU. Alguns fatores, portanto, possibilitam o surgimento dessa infecção, como: sexo feminino, idade avançada e o tempo de permanência do cateter. 
(Campos et. al., 2016; Anjos, et al. 2020).

Praticamente, todas as investigações têm demonstrado que o sexo feminino apresenta as mais altas taxas de bacteriúria associada ao cateter ao sexo masculino. Igualmente, a idade avançada e a coexistência de uma doença grave em pacientes cateterizados e hospitalizados, constituem importantes fatores de risco, que proporcionam, inclusive, um aumento da taxa de mortalidade. (Lenz; Lino \& Lima, 2006)

Através dos trechos abordados acima, percebe-se que ao realizar o procedimento de cateterismo vesical de demora, o enfermeiro deverá estar atento aos fatores de risco e outras possíveis patologias que o paciente pode apresentar, de modo a evitar o surgimento de infecção do trato urinário e o agravamento do quadro clínico, debilitando ainda mais o cliente.

\section{Conclusão}

Em suma, a infecção do trato urinário consiste em um importante problema de saúde pública na atualidade. Dessa forma, a doença pode ser elencada como uma das infecções relevantes que podem ser contraídas pelo paciente dentro do ambiente hospitalar.

Um dos procedimentos realizados em larga escala em unidades hospitalares, representa-se pelo cateterismo vesical de demora. Um cuidado importante que tende a auxiliar o paciente a esvaziar a bexiga quando necessário. Entretanto, esse procedimento pode acarretar riscos e danos à saúde dos pacientes, quando não realizado de forma criteriosa e asséptica.

Os resultados dessa pesquisa permitem concluir que, o tempo de permanência do cateter no sistema urinário contribui consideravelmente para o surgimento de sinais e sintomas de infecção urinária após o cateterismo vesical de demora. E, quanto maior o tempo do cateter no organismo do paciente, maior o risco do desenvolvimento dessa infecção.

Ademais, fez-se plausível concluir que, são altos os índices de infecção urinária por cateterização vesical de demora em Unidades de Terapia Intensiva, pois, os pacientes que se encontram ali internados apresentam maiores necessidades de saúde por estarem criticamente enfermos. Os enfermeiros necessitam em todos os cenários hospitalares estarem preparados técnico-cientificamente para a realização desse cuidado junto ao paciente e, treinar a sua equipe para tal.

Reitera-se, que os fatores de risco influenciam consideravelmente no desenvolvimento dos sinais e sintomas de infecção em pacientes que foram submetidos ao cateterismo vesical de demora. Os pacientes que apresentam esses fatores de risco necessitam de ser monitorizados cuidadosamente pela equipe de enfermagem.

Cabe aos enfermeiros e equipe sob sua supervisão estar continuamente discutindo a prática do cateterismo vesical de demora em ambiente hospitalar, seus riscos, e a possibilidade de execução da técnica visando a segurança do paciente, seu bem-estar e diminuição do tempo de permanência hospitalar.

Sugere-se novas pesquisas que identifiquem in loco a associação entre fatores de risco modificáveis e não modificáveis e o surgimento de infecções do trato urinário após a instalação de cateter vesical de demora.

\section{Referências}

Anjos, M. K. dos, Azevedo, T. G. L. D`Eça Junior, A., Silva, R. A. R. Stipp, M. A. C., \& Paes, G. O. (2020). Incidentes associados ao cateterismo vesical de demora em pacientes hospitalizados: revisão integrativa. Research, Society and Development, 9(8), e41985115. https://doi.org/10.33448/rsd-v9i8.5115

Amaral, D. M. do et al. (2017). Pós-operatório de Vulvectomia e Cateterismo Vertical de Demora: Revisão Integrativa. Revista de Enfermagem. REVOL,11(10), 3948-3957.

Arrais, E. L. M.; Oliveira, M, L, C. \& Souza, I, D, B. (2017) Prevenção de Infecção Urinária: Indicadores de Qualidade da Assistência de Enfermagem em Idoso. UFPE, 11(8), 3151-3157.

Campos, C. C. et al. (2016) Incidência de Infecção do Trato Urinário Relacionada ao Cateterismo Vesical de Demora: Um Estudo de Corte. Reme, 20(903).

Cardoso, S. A. C, \& Maia, L. F. S. (2014). Cateterismo Vesical de Demora na UTI Adulto: O Papel do Enfermeiro na Prevenção de Infecção do Trato Urinário. Recien, 4(12), 5-14.

Cavalcante, T. M. C.; Braguehais, A. R. \& Bezerra, P. A. R. G. (2017) Sonda Vesical de Demora: Perfil Epidemiológico da Infecção Urinária no Centro de Terapia Intensiva. Tendência da Enfermagem Profissional. 9(2), 2164-2169 
Chaer, P. G.; Diniz, P. R. R. P, \& Ribeiro, P. D. E. A. (2011) A técnica do Questionário na Pesquisa Educacional. Evidência 7(7), $251-266$.

Chaves, N. M. O, \& Moraes, C. L. K. (2015) Controle de Infecção em Cateterismo Vesical de Demora em Unidade de Terapia Intensiva. RECOM. 5(2), 16501657.

Ercole, F. F.; Macieira, T. G. R.; Wenceslau, L. C. C.; Martins, A. R.; Campos, C. C, \& Chiana, T. C. M. (2013) Revisão Integrativa: Evidências na Prática do Cateterismo Urinário Intermitente/Demora. Revista Latino - Am Enfermagem. 21(1), 1-10

Farias, R. C.; Nascimento, C. C. L. \& Souza, M. W. O. (2019) Infecções do Trato Urinário Relacionado ao Cateter Vesical de Demora: Elaboração de Bundle. Eletrônica Acervo Saúde. 11(11).

Godoy, S.; Marchi-Alves, L. M, \& Larchercaliri, M. H. (2015). Aprender Para Cuidar em Enfermagem: Situações Especificas de Aprendizagem: cateterismo vesical de demora masculino e feminino. Escola de Enfermagem de Ribeirão Preto- USP. 60.

Hinkle, J, \& Cheever, K. (2015) Tratado de Enfermagem Médico-Cirúrgica. Guanabara Koogan.

Jesus, J. S.; Coelho, M. F, \& Luz, R. A. (2018) Cuidados de Enfermagem Para Prevenção de Infecção do Trato Urinário em Pacientes com Cateterismo Vesical de Demora (CVD) no Ambiente Hospitalar. Arquivos Médicos. 63(2), 96-99.

Jorge, Beatriz Maria, \& Mazzo, Alessandra, \& Costa Mendes, Isabel Amélia, \& Trevizan, Maria Auxiliadora, \& Martins, José Carlos Amado (2013). Infeção do trato urinário relacionada com o uso do cateter: revisão integrativa. Revista de Enfermagem Referência, III (11),125-132.[fecha de Consulta 24 de Junio de 2021]. ISSN: 0874-0283. https://www.redalyc.org/articulo.oa?id=388239970011

Magalhaes, S. R.; Melo, E. M.; Lopes V. P.; Carvalho, Z. M. F.; Barbosa, V. I.; Studart. R. M. B. (2014) Evidências Para a Prevenção de Infecção no Cateterismo Vesical: Revisão Integrativa. JNUOL. 8(4), 1057-1063.

Marks, F. O., Oliveira, T. M. S. de, Ferreira, G., Dallabrida, M. M., Bisewski, C. G., \& Souza, P. A. de. (2020). Infecção do trato urinário: etiologia, perfil de sensibilidade e resistência aos antimicrobianos em hospital pediátrico. Research, Society and Development, 9(8), e677985807. https://doi.org/10.33448/rsdv9i8.5807

Mazzo, A.; Godoy, S.; Alves, L, M.; Mendes, I. A. C.; Trevizan, M. A. \& Rangel, E. M. L. (2011) Cateterismo Urinário: Facilidades e Dificuldades Relacionadas à Sua Padronização. Texto \& Contexto Enfermagem. 20(2), 333-339.

Menegueti, M. G.; Auxiliadora, M. M.; Marin, R. S. C. S.; Basile, A. F. \& Laus, A. M. (2012) Infecção Urinária em Unidade de Terapia Intensiva: um Indicador de Processo para Prevenção. RENE. 13(3), 632-638.

Michel, M. H. (2015) Metodologia e Pesquisa Científica e Ciências Sociais. Atlas.

Mota, É. C. \& Oliveira, A. C. (2019) Infecção do Trato Urinário Associado a Cateter Vesical: Por Que Não Controlamos Esse Evento Adverso. Escola de Enfermagem da USP. 53, 3452-3452.

Perry, A. G. (2015) Guia Completo de Procedimentos e Competências de Enfermagem. Guanabara Saúde Didático.

Ramalho, A. \& Matta, A. C. G. (2017) Incidência de Infeççao urinária entre receptores de Transplante Renal. Acta Scientiarum. Health Sciences. 39(2), 183188 .

Rodrigues, F. J. B. \& Barroso, A. P. D. (2011) Etiologia e Sensibilidade Bacteriana em Infecções do Tracto Urinário. Portuguesa de Saúde Pública. 29(2), $123-131$.

Santo, F. H. E. (2008) Infecção do trato Urinário: Pesquisando Evidências Para o Cuidado de Enfermagem. Online Brazilian Journal of Nursing.7(3).

Souza, A. C. S.; Tipple, A. F. V.; Barbosa, J. M.; Pereira, M. S. \& Barreto, R. A. S. S. (2007) Cateterismo Urinário: Conhecimento e Adesão ao Controle de Infecção Pelos Profissionais de Enfermagem. Eletrônica de Enfermagem. 09(03), 724-735.

Souza, A. C. S. e et al. (2016) Infeções do Trato Urinário e Outras Infecções do Sistema Urinário: medidas de prevenção de infecções relacionadas à assistência à saúde. ANVISA.

Stacciarini, T. S. G. (2014) Plano de Intervenções em Enfermagem: Prevenção de Eventos Adversos Relacionados ao Cateter Vesical de Demora. Universidade Federal do Triângulo Mineiro.

Stamm, A. M. N. F. \& Coutinho, M. S. S. A. (1999) Infecção do Trato Urinário Relacionada ao Cateter Vesical de Demora: Incidência e Fatores de Risco. Revista da Associação Médica Brasileira. 45(1), 27-33.

Trindade, J. S. ., Silva, E. G. da ., Furtado, G. de S. ., Carvalho, D. de N. R. de ., Bendelaque, D. de F. R. ., Costa, R. E. A. R. da ., Albuquerque, L. P. ., Silva, K. S. O. e ., Rodrigues, R. de S. ., Pinheiro, A. C. M. ., Pantoja, A. C. R. ., Vaz, G. L. ., Santos, C. C. dos ., Aguiar, V. F. F. de ., Nogueira, M. de A. ., \& Sá, A. M. M. . (2020). Infecção relacionada à assistência à saúde: Prevalência em Unidade de Terapia Intensiva Adulto. Research, Society and Development, 9(9), e373997107. https://doi.org/10.33448/rsd-v9i9.7107

Vieira, F. A. (2009) Ações de Enfermagem Para Prevenção de Infecção do Trato Urinário Relacionada ao Cateter Vesical de Demora. Eistein. 7(3), 21153216.

Zanella, L. C. H. (2009) Metodologia de Estudo e de Pesquisa em Administração. Capes Mec. 